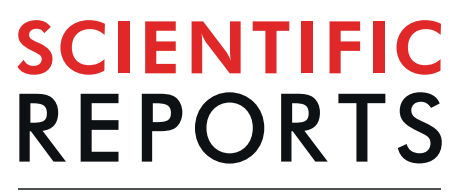

natureresearch

\title{
Association between Serum Gamma-glutamyl transferase and Intracranial Arterial Calcification in Acute Ischemic Stroke Subjects
}

\author{
Tao Yao $\mathbb{1}^{1}$, Jing Li ${ }^{2,3}$, Qi Long ${ }^{2,3}$, Gang Li $\mathbb{1}^{2,3^{*}}$, Yanbin Ding ${ }^{4 *}$, Qin Cui ${ }^{1}$ \& Zhichao Liu ${ }^{1}$
}

Intracranial artery calcification (IAC) is an important risk factor for cerebral infarction and a key biomarker for intracranial artery stenosis. Gamma-glutamyl transferase (GGT) has been independently associated with increased cardiovascular events and coronary calcification. Our study assessed whether GGT is an independent factor for IAC in acute ischemic stroke (AIS) patients. This cross-sectional study involved a total of 754 patients with AIS (mean age: $65 \pm 13.2$ years). All the patients had received brain computed tomography angiography (CTA) examination to evaluate IAC. Further, serum GGT levels and other biochemical parameters were analyzed. The average GGT level in patients who died was also significantly increased ( $37.0 \pm 26.8$ vs $29.0 \pm 21.5 \mathrm{U} / \mathrm{L}, \mathrm{p}=0.012$ ). Partial correlation analysis showed that serum GGT levels were associated with NIHSS score at admission after adjustment for age and gender was considered $(r=0.150, p=0.001)$. Logistic regression analysis showed that serum GGT levels independently predicted all-cause mortality (OR $=1.036,95 \% \mathrm{Cl}: 1.014-1.060, \mathrm{p}=0.002)$, NIHSS scores $(\beta=0.051,95 \% \mathrm{Cl}: 0.020-0.082, p=0.001)$ and IAC scores $(\beta=0.006,95 \% \mathrm{Cl}: 0.003-0.014, p=0.005)$ in male patients. Each SD (standard deviation) increase of serum GGT levels was also associated with risk of all-cause mortality ( $\mathrm{OR}=2.272,95 \% \mathrm{Cl}: 1.364-3.787, P=0.002)$. GGT levels in patients with severe IAC were significantly elevated $(37.6 \pm 33.6$ vs $28.6 \pm 19.2, p<0.001)$. However, serum GGT levels could not independently predict the severity of IAC in AIS patients. Our study identified that serum GGT levels were significantly elevated in patients who died, and the GGT levels had a certain association with the risk of death and IAC in male patients.

Intracranial artery calcification (IAC) can be easily identified through a computed tomography (CT) head scan, thus enabling it as a potential non-invasive biomarker. A large number of accumulated evidences in patients, especially in Asian population, suggest a correlation between IAC and ischemic stroke, decreased cognitive ability, and other vascular diseases ${ }^{1-3}$. IAC is an important risk factor for cerebral infarction and a key biomarker for intracranial artery stenosis.

The degree of IAC is related to the condition of atheromatous plaques. The coronary calcification score is currently widely used to assess the risk of cardiovascular events in patients. Previously, we found that serum gamma-glutamyl transferase (GGT) levels were independently associated with progression of coronary calcification in patients with type 2 diabetes mellitus (T2DM $)^{4}$. GGT is a key enzyme that hydrolyzes the antioxidant glutathione. This hydrolysis reaction produces many pro-oxidants including reactive oxygen species (ROS) and oxidized low-density lipoprotein (OX-LDL), which can increase the formation and development of atherosclerotic plaques ${ }^{5}$. Previous studies found that blood GGT activity were independent biomarkers of coronary calcification $^{6,7}$. However, the relationship between GGT and IAC has not been further evaluated.

A large number of studies have shown that GGT could be used as a risk factor for cardiovascular diseases (CVD) and could allow to independently predict all-cause mortality and fatal cardiovascular events in patients with coronary heart diseases (CHD ${ }^{8,9}$. Jousilahti et al., reported that the GGT enzyme is associated with ischemic stroke $^{10}$. A study with a small sample size showed that elevated GGT levels were associated with infarct size in

${ }^{1}$ Department of Neurology, Renmin Hospital of Wuhan University, Wuhan, China. ${ }^{2}$ Emergency Department, Hubei Provincial Hospital of Traditional Chinese Medicine, Wuhan, 430061, China. ${ }^{3}$ Hubei Province Academy of Traditional Chinese Medicine, Wuhan, China. ${ }^{4}$ Department of Neurology, Hubei Provincial Hospital of Traditional Chinese Medicine, Wuhan, 430061, China. *email: marty007@163.com; 13886058258@163.com 
acute ischemic stroke (AIS) ${ }^{11}$. Furthermore, Tu W. J. et al., demonstrated that GGT was independently associated with all-cause and CVD mortality in patients with ischemic stroke ${ }^{12}$. In this study, the main objectives are to assess the relationship between GGT and IAC and to assess whether GGT levels can independently predict short-term mortality in patients with AIS.

\section{Methods}

Subjects. The present study was performed in Department of Neurology, Renmin Hospital, Wuhan University, China. All patients referred for AIS between January 2017 and December 2018 were screened. Each patient received brain CT and/or MR imaging and the diagnosis of ischemic stroke was based on the agreement of at least two neurologists. The present study was in line with the Declaration of Helsinki. This study was approved by the Human Research Ethics Committee of Renmin Hospital of Wuhan University (Wuhan, China, No. 2016H11018). Each participant received written informed consent. We have signed the consent of each study participant. All results were anonymous. No separate results are displayed, only group statistics are provided.

According to AHA/ASA stroke guidelines, 913 adult patients above 35 years of age with initially AIS were admitted to the University Affiliated Hospital within three days of onset of symptoms. Patients with a history of stroke or old cerebral infarction on CT/MRI images were excluded from the initial screening. In this study, we used the WHO definition to determine whether patients were smoking or drinking. Smoking is defined as smoking more than one cigarette a day for six months. Drinking is defined as drinking at least once in the past 30 days. These information were obtained directly from study patients or their hospital medical records.159 patients were excluded from the study: 30 patients did not undergo computed tomography angiography (CTA) examination, 63 patients were missing follow-up results, 31 patients had a history of chronic liver disease and significant abnormal liver function, and 35 patients did not receive National Institutes of Health Stroke Scale (NIHSS) score at admission. Therefore, a total of 754 patients were finally included in this cross-sectional study.

Biochemical measurements. All participants included in the study had accepted head CT, electrocardiogram (ECG), magnetic resonance imaging (MRI), transthoracic echocardiography (TEE) and standard laboratory examinations at admission. Blood samples were collected from the antecubital vein in a vacuum tube containing EDTA. Patients were asked to fast for 8 hours, and levels of liver enzyme, blood lipid profiles, renal biochemical index, fasting plasma glucose (FPG), homocysteine (Hcys) and glycosylated hemoglobin (HbA1c) levels were measured by standard methods. Serum GGT activity was analyzed with an enzyme kinetic assay (Olympus AU600; Roche Diagnostics, Shanghai, China), based on the recommendation of European Committee for Clinical Laboratory Standards ${ }^{13}$. Serum GGT activity was measured at $37^{\circ} \mathrm{C}$ and recalculated to $25^{\circ} \mathrm{C}$ and were expressed as units per liter $(\mathrm{U} / \mathrm{L})$. Between-analyzer differences were $<5 \%$. The reference range for GGT activity was 0 to $50 \mathrm{U} / \mathrm{L}^{13}$. The following variables were collected in the AIS phase: age, gender, causes of ischemic stroke (according to TOAST criteria $)^{14}$, previously confirmed or found risk factors such as hypertension, diabetes, BMI, coronary heart disease (CHD), peripheral artery disease (PAD), smoking, and long-term alcohol consumption.

Assessment of IAC score. All patients underwent multidetector brain CTA using a 64-slice spiral CT device (GE Healthcare, Milwaukee, WI, USA). The parameters were as follows: $120 \mathrm{kVp}, 140 \mathrm{~mA}, 0.9-\mathrm{mm}$ section thickness, $0.9-\mathrm{mm}$ slice acquisition interval, and intravenous administration of $80 \mathrm{~mL}$ of iohexol at a rate of $5.0 \mathrm{~mL} / \mathrm{s}^{15}$. Bone window CT images covered the whole brain (from the skull base to the vertex), to identify the presence of IAC. IAC lesions were defined as high density lesions with a median density greater than 130 Hounsfield units ${ }^{16}$. We used the 5-point scale semi-quantitative scoring system proposed by Babiarz et al. to evaluate IAC ${ }^{17}$. We selected the highest calcification score for each cerebral artery and added the calcification score of all evaluated artery to get the patient's total calcification score. We distinguished the severity of IAC according to the total calcification score. 0 score for absent, 1-4 for mild, 5-8 for moderate, and 9-12 for severe degree ${ }^{18}$. Two experienced neurologists independently reviewed the images from CT angiography and graded the degree of IAC.

Statistical analyses. We used SPSS version 22.0 (SPSS Inc, Chicago, Illinois) statistical software for statistical analysis. Comparison of continuous variables in normal distribution by Student's t-test and ANOVA. A $\log _{2}$ transformation of non-normal distributed variables was used to reduce the influence of skew. The categorical variables were analyzed by chi-squared test. Patients were divided into four quartile groups according to the serum GGT levels. Adjusted for age and gender, Partial Spearman correlation coefficients were used to clarify the association between serum GGT with Hcys and NIHSS score. We used logistic regression analysis to examine the relationships between serum GGT levels with all-cause hospital mortality risk, NIHSS score and IAC score. These unified variables of age, hypertension, diabetes mellitus, smoking, drinking, CHD, DBP, TC, TG, Hcys, HbA1c and uric acid (UA) were adjusted in logistic regression analyses. Further analyses were performed to determine multivariable-adjusted odds ratios (ORs) of all-cause hospital mortality risk and severe IAC for the quartiles of serum GGT levels in male AIS patients. In additional, $\log$ transformed of GGT was analyzed for $P_{\text {trend }}$. $Z$ scores for GGT standardization was analyzed for Per 1 SD (standard deviation) increase of ORs. $P<0.05$ was considered to have significant difference.

\section{Results}

A total of 754 AIS patients with an average age of $65 \pm 13.2$ years, including 515 males were recruited in the study. We reviewed the 60-day follow-up of patients after admission and found that 85 patients died during hospitalization and follow-up. The median GGT level included in the study was $23.0(16.0-36.0) \mathrm{U} / \mathrm{L}$. The mean IAC score was $3.77 \pm 3.50$. The mean admission NIHSS score was $6.55 \pm 6.34$. According to TOAST classification, 284 patients were grouped into type 1 infarction, 313 patients were type 2, 63 patients were type 3,76 patients were type 4 and 18 patients were type 5 . Analysis of variance showed that the NIHSS score and mortality rate of the patients in the highest GGT quantile group were significantly higher than those of the other three groups 


\begin{tabular}{|c|c|c|c|c|c|}
\hline & $\begin{array}{l}\text { Quartile } 1 \\
\leq 16 \mathrm{U} / \mathrm{L}\end{array}$ & $\begin{array}{l}\text { Quartile 2 } \\
\text { 17-23 U/L }\end{array}$ & $\begin{array}{l}\text { Quartile } 3 \\
\text { 24-36 U/L }\end{array}$ & $\begin{array}{l}\text { Quartile 4 } \\
\geq 37 \mathrm{U} / \mathrm{L}\end{array}$ & $P$ value \\
\hline Age (years) & $69.7 \pm 13.4$ & $66.4 \pm 11.5$ & $63.7 \pm 12.7$ & $61.1 \pm 13.7$ & $<0.001$ \\
\hline Male, n (\%) & $94(49.6 \%)$ & $134(71.4 \%)$ & $145(76.8 \%)$ & $142(75.4 \%)$ & $<0.001$ \\
\hline $\mathrm{BMI}\left(\mathrm{kg} / \mathrm{m}^{2}\right)$ & $22.85 \pm 2.59$ & $22.81 \pm 2.67$ & $23.78 \pm 2.71$ & $23.92 \pm 2.94$ & $<0.001$ \\
\hline Smoking, n (\%) & $42(22.3 \%)$ & $73(38.9 \%)$ & $80(42.7 \%)$ & 77 (40.7\%) & 0.004 \\
\hline Drinking, n (\%) & $23(12.4 \%)$ & $48(25.4 \%)$ & $51(27.0 \%)$ & $48(25.4 \%)$ & 0.023 \\
\hline HT, n (\%) & $126(66.9 \%)$ & $135(71.4 \%)$ & $131(69.6 \%)$ & $129(68.6 \%)$ & 0.894 \\
\hline $\mathrm{DM}, \mathrm{n}(\%)$ & $50(26.7 \%)$ & $49(26.2 \%)$ & $66(34.8 \%)$ & $67(35.6 \%)$ & 0.226 \\
\hline CHD, n (\%) & $23(12.4 \%)$ & $15(7.9 \%)$ & $20(10.7 \%)$ & $22(11.9 \%)$ & 0.606 \\
\hline SBP, $\mathrm{mmHg}$ & $149.0 \pm 25.1$ & $153.2 \pm 26.9$ & $147.6 \pm 25.5$ & $150.9 \pm 26.8$ & 0.370 \\
\hline $\mathrm{DBP}, \mathrm{mmHg}$ & $82.4 \pm 13.9$ & $83.6 \pm 14.7$ & $84.8 \pm 15.7$ & $89.2 \pm 16.3$ & 0.004 \\
\hline HR (bpm) & $76.8 \pm 13.2$ & $74.8 \pm 11.5$ & $79.0 \pm 13.4$ & $80.2 \pm 15.2$ & 0.01 \\
\hline ALT (U/L) & $14.9 \pm 8.7$ & $17.0 \pm 7.1$ & $21.0 \pm 9.5$ & $26.2 \pm 16.2$ & $<0.001$ \\
\hline AST (U/L) & $21.2 \pm 8.8$ & $19.6 \pm 5.6$ & $22.2 \pm 9.0$ & $27.7 \pm 14.8$ & $<0.001$ \\
\hline $\mathrm{Cr}(\mathrm{umol} / \mathrm{L})$ & $75.3 \pm 28.6$ & $77.6 \pm 34.8$ & $75.3 \pm 25.9$ & $77.5 \pm 26.2$ & 0.883 \\
\hline UA (umol/L) & $343.6 \pm 101.0$ & $371.7 \pm 124.9$ & $379.1 \pm 102.2$ & $385.5 \pm 135.2$ & 0.032 \\
\hline FBG (mmol/L) & $6.6 \pm 3.1$ & $6.8 \pm 3.5$ & $7.5 \pm 3.7$ & $7.9 \pm 3.8$ & 0.019 \\
\hline $\mathrm{TC}(\mathrm{mmol} / \mathrm{L})$ & $4.20 \pm 0.93$ & $4.50 \pm 1.00$ & $4.59 \pm 1.13$ & $4.68 \pm 1.07$ & 0.004 \\
\hline $\mathrm{TG}(\mathrm{mmol} / \mathrm{L})$ & $1.39 \pm 0.75$ & $1.49 \pm 0.75$ & $1.87 \pm 1.58$ & $2.05 \pm 1.75$ & $<0.001$ \\
\hline $\mathrm{HDL}-\mathrm{C}(\mathrm{mmol} / \mathrm{L})$ & $1.12 \pm 0.38$ & $1.13 \pm 0.45$ & $1.08 \pm 0.33$ & $1.18 \pm 0.99$ & 0.619 \\
\hline LDL-C (mmol/L) & $2.36 \pm 0.76$ & $2.65 \pm 0.81$ & $2.62 \pm 0.96$ & $2.65 \pm 0.87$ & 0.028 \\
\hline sdLDL-C (mmol/L) & $0.73 \pm 0.31$ & $0.89 \pm 0.41$ & $1.05 \pm 0.56$ & $0.98 \pm 0.43$ & $<0.001$ \\
\hline ApoA1 (g/L) & $1.29 \pm 0.21$ & $1.30 \pm 0.20$ & $1.33 \pm 0.22$ & $1.33 \pm 0.26$ & 0.424 \\
\hline ApoB (g/L) & $0.86 \pm 0.29$ & $0.90 \pm 0.23$ & $0.94 \pm 0.22$ & $0.95 \pm 0.23$ & 0.063 \\
\hline HbAlc (\%) & $6.44 \pm 1.51$ & $6.90 \pm 1.90$ & $7.13 \pm 1.86$ & $7.14 \pm 1.91$ & 0.033 \\
\hline D-dimer (mg/L) & $1.35 \pm 2.06$ & $0.95 \pm 1.90$ & $0.99 \pm 1.92$ & $1.70 \pm 3.18$ & 0.06 \\
\hline Hcys (umol/L) & $19.0 \pm 12.6$ & $18.6 \pm 11.7$ & $18.0 \pm 8.7$ & $20.8 \pm 15.5$ & 0.423 \\
\hline IAC score & $3.41 \pm 3.46$ & $3.67 \pm 3.35$ & $3.79 \pm 3.51$ & $4.21 \pm 3.68$ & 0.358 \\
\hline Death, n (\%) & $15(8.3 \%)$ & $12(7.1 \%)$ & $21(11.6 \%)$ & $37(20.3 \%)$ & 0.006 \\
\hline NIHSS score & $5.75 \pm 5.42$ & $5.93 \pm 6.28$ & $6.35 \pm 6.61$ & $8.24 \pm 6.78$ & 0.009 \\
\hline
\end{tabular}

Table 1. Clinical and laboratory data for 754 patients divided into quartiles of serum GGT levels. ALT, alanine aminotransferase; AST, aspartate aminotransferase; BMI, body mass index; SBP, systolic blood pressure; DBP, dilated blood pressure; HbA1c, glycated hemoglobin; LDL-C, low-density lipoprotein cholesterol; HDL-C, high-density lipoprotein cholesterol; TC, total cholesterol; TG, total triglyceride FPG, fasting plasma glucose; GGT, gamma-glutamyltransferase; UA, uric acid; BUN, blood urea nitrogen; Apo B: Apolipoproteins B; ApoA1: Apolipoproteins A-1; sdLDL-C: Small dense low-density lipoprotein cholesterol; HT, hypertension; Hcys, homocysteine; IAC, intracranial artery calcification; NIHSS, National Institutes of Health Stroke Scale.

(Table 1). The average GGT level in patients who died was also significantly higher than that of the survived patients $(37.0 \pm 26.8$ vs $29.0 \pm 21.5 \mathrm{U} / \mathrm{L}, \mathrm{p}=0.012$, Fig. 1$)$. However, there were no significant differences in IAC scores between patients in different quantile groups (Table 1).

We analyzed the association between the GGT level to the risk factor of Hcys and the NIHSS score reflecting the prognosis of stroke patients. After adjusting age and gender, partial correlation analysis showed that there was no significant correlation between GGT and Hcys $(r=0.025, p=0.618$, Fig. 2), whereas there was a certain correlation with the NIHSS score $(r=0.150, p=0.001$, Fig. 2$)$.

Logistic regression analysis showed that serum GGT levels predicted short-term all-cause mortality $(\mathrm{OR}=1.016,95 \% \mathrm{CI}: 1.004-1.029, \mathrm{p}=0.012)$, NIHSS scores $(\beta=0.035,95 \% \mathrm{CI}: 0.006-0.064, \mathrm{p}=0.017)$, and IAC scores $(\beta=0.009,95 \%$ CI: $0.002-0.015, p=0.008)$ in male patients with unadjusted variables (Table 2$)$. After adjustment of age, smoking, drinking, HT, DM, CHD, DBP, TC, TG, Hcys, HbA1c and UA, GGT levels could independently predict the risk of all-cause mortality $(\mathrm{OR}=1.036,95 \% \mathrm{CI}: 1.014-1.060, \mathrm{p}=0.002)$, NIHSS scores $(\beta=0.051,95 \%$ CI: $0.020-0.082, p=0.001)$ and IAC scores $(\beta=0.006,95 \%$ CI: $0.003-0.014, p=0.005)$ in male patients. However, GGT levels could not independently be associated with all-cause mortality, NIHSS scores and IAC scores in female patients (Table 2).

Table 3 shows the relationships between male patient's serum GGT quartiles and all-cause hospital mortality both in unadjusted and adjusted models. Multivariable logistic regression analysis showed that the risk of all-cause hospital mortality in fourth GGT quartile group was significantly higher than other quartiles, both in univariate analysis $\left(\mathrm{OR}=4.164,95 \% \mathrm{CI}\right.$ : $\left.1.157-14.991, P=0.029, P_{\text {trend }}=0.031\right)$ and after adjustment for the 12 demographic, medical history and laboratory variables $(\mathrm{OR}=5.616,95 \% \mathrm{CI}: 1.339-20.641, P=0.034$, $\left.P_{\text {trend }}=0.034\right)$. Each SD increase of serum GGT levels (Z score transformed) was also associated with risk of 


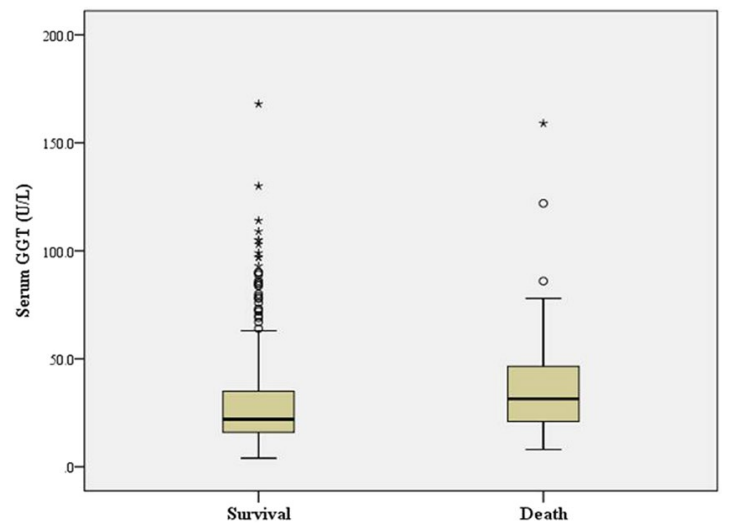

Figure 1. Box and whisker plots illustrate the mean and interquartile ranges of serum GGT levels in patients with survival and death. Open circles and asterisks show outliers.
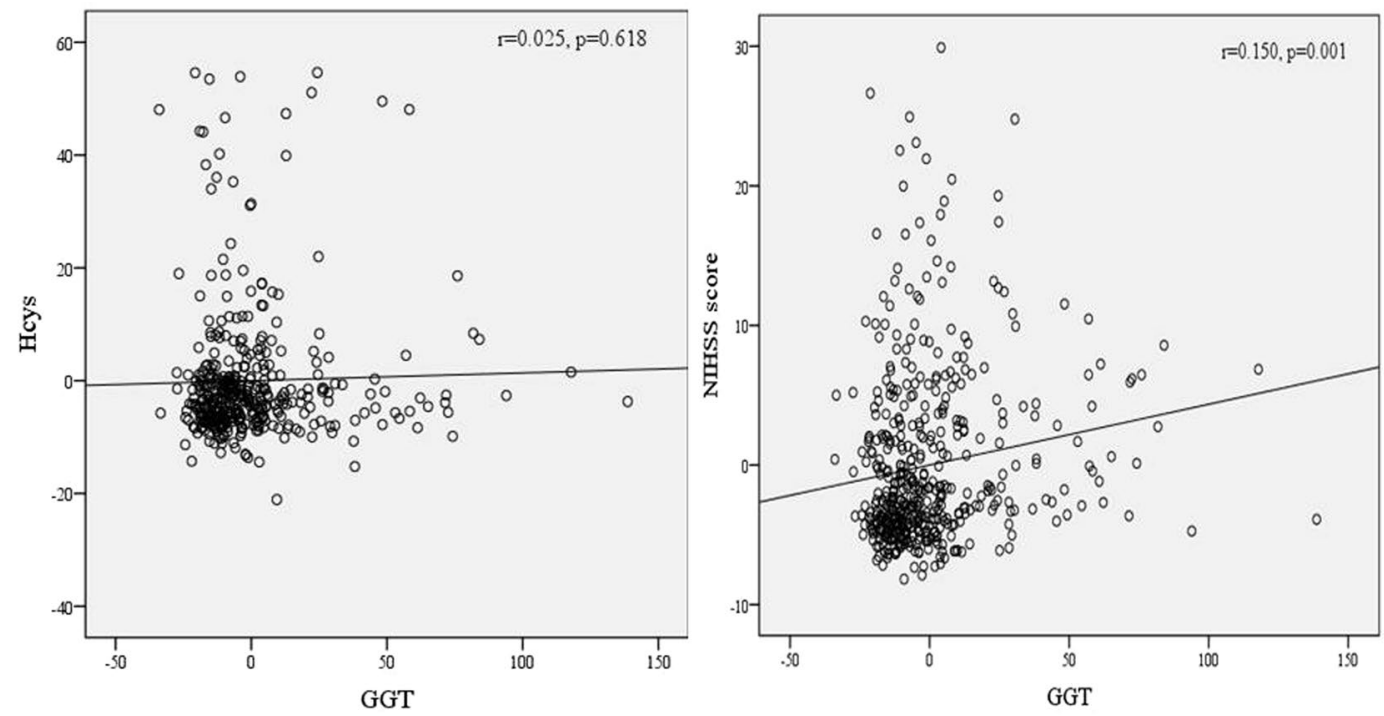

Figure 2. Partial Spearman correlation coefficients showed the association between serum GGT levels with serum homocysteine levels and NIHSS score after adjustment for age and gender.

\begin{tabular}{|l|l|l|l|l|l|l|l|l|l|l|}
\hline \multirow{2}{*}{ Male } & Death & NIHSS score & \multicolumn{1}{l|}{ IAC score } \\
\cline { 2 - 11 } & OR & $\mathbf{9 5 \%}$ CI & P value & $\boldsymbol{\beta}$ & $\mathbf{9 5 \%}$ CI & P value & $\boldsymbol{\beta}$ & 95\% CI & $P$ value \\
\hline unadjusted & 1.016 & $1.004-1.029$ & 0.012 & 0.035 & $0.006-0.064$ & 0.017 & 0.009 & $0.002-0.015$ & 0.008 \\
\hline Model 1 & 1.020 & $1.006-1.034$ & 0.004 & 0.042 & $0.012-0.072$ & 0.006 & 0.008 & $0.001-0.015$ & 0.017 \\
\hline Model 2 & 1.036 & $1.014-1.060$ & 0.002 & 0.051 & $0.020-0.082$ & 0.001 & 0.006 & $0.003-0.014$ & 0.005 \\
\hline Female \\
\hline unadjusted & 1.010 & $0.991-1.029$ & 0.300 & 0.048 & $-0.006-0.101$ & 0.080 & 0.008 & $-0.003-0.019$ & 0.145 \\
\hline Model 1 & 1.015 & $0.994-1.036$ & 0.157 & 0.060 & $0.007-0.114$ & 0.027 & 0.007 & $-0.005-0.018$ & 0.257 \\
\hline Model 2 & 0.752 & $0.524-1.078$ & 0.121 & 0.036 & $-0.071-0.072$ & 0.991 & 0.015 & $-0.003-0.033$ & 0.110 \\
\hline
\end{tabular}

Table 2. Association of serum GGT levels with death, NIHSS score and IAC score according to univariate and multivariate analyses. Model 1 adjusted for age, smoking, drinking, HT, DM, CHD; Model 2 adjusted for Model 1 plus DBP, TC, TG, Hcys, HbA1c and UA.

all-cause hospital mortality, both in univariate analysis $(\mathrm{OR}=1.443,95 \% \mathrm{CI}: 1.085-1.919, P=0.012)$ and after adjustment $(\mathrm{OR}=2.272$, 95\% CI: $1.364-3.787, P=0.002)$

A total of 117 patients were diagnosed with severe IAC. GGT levels in patients with severe IAC were significantly elevated $(37.6 \pm 33.6$ vs $28.6 \pm 19.2, \mathrm{p}<0.001)$. However, NIHSS scores $(6.66 \pm 5.78$ vs $6.53 \pm 6.44$, $\mathrm{p}=0.873)$ and mortality $(8.1 \%$ vs $12.4 \%, \mathrm{p}=0.333)$ in patients with severe IAC were not significantly different from those in patients with non-severe IAC. Logistic regression analysis showed that after adjusting 12 variables, 


\begin{tabular}{|c|c|c|c|c|c|c|}
\hline Death & Quartile 1 & Quartile 2 & Quartile 3 & Quartile 4 & $P_{\text {trend }}$ & Each SD increase of GGT \\
\hline Unadjusted & \multirow{2}{*}{1} & $1.118(0.257-4.862)$ & $2.500(0.658-9.501)$ & $4.164(1.157-14.991)$ & \multirow{2}{*}{0.031} & $1.443(1.085-1.919)$ \\
\hline$P$ values & & 0.882 & 0.179 & 0.029 & & 0.012 \\
\hline Model 1 & \multirow{2}{*}{1} & $1.252(0.278-5.643)$ & $2.952(0.745-11.698)$ & $4.785(1.261-18.156)$ & \multirow{2}{*}{0.028} & $1.589(1.157-2.182)$ \\
\hline$P$ values & & 0.770 & 0.123 & 0.021 & & 0.004 \\
\hline Model 2 & \multirow{2}{*}{ 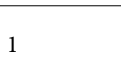 } & $0.895(0.046-7.432)$ & $3.057(0.849-14.156)$ & $5.616(1.339-20.641)$ & \multirow{2}{*}{0.034} & $2.272(1.364-3.787)$ \\
\hline$P$ values & & 0.941 & 0.153 & 0.034 & & 0.002 \\
\hline
\end{tabular}

Table 3. Odds ratios and $95 \%$ confidence intervals of all-cause hospital mortality risk according to serum GGT quartiles in male AIS patients. Model 1 adjusted for age, smoking, drinking, HT, DM, CHD; Model 2 adjusted for Model 1 plus DBP, TC, TG, Hcys, HbAlc and UA.

\begin{tabular}{|c|c|c|c|c|c|c|}
\hline Several IAC & Quartile 1 & Quartile 2 & Quartile 3 & Quartile 4 & $P_{\text {trend }}$ & Each SD increase of GGT \\
\hline Unadjusted & \multirow{2}{*}{1} & $0.548(0.188-1.601)$ & $1.486(0.591-3.733)$ & $1.884(0.770-4.613)$ & \multirow{2}{*}{0.054} & $1.409(1.091-1.820)$ \\
\hline$P$ values & & 0.272 & 0.400 & 0.166 & & 0.009 \\
\hline Model 1 & \multirow{2}{*}{1} & $0.514(0.174-1.521)$ & $1.365(0.534-3.489)$ & $1.695(0.676-4.252)$ & \multirow{2}{*}{0.082} & $1.386(0.061-1.810)$ \\
\hline$P$ values & & 0.229 & 0.516 & 0.261 & & 0.017 \\
\hline Model 2 & \multirow{2}{*}{1} & $0.815(0.209-3.176)$ & $1.952(0.604-6.305)$ & $1.874(0.555-6.321)$ & \multirow{2}{*}{0.366} & $1.230(0.871-1.735)$ \\
\hline$P$ values & & 0.768 & 0.263 & 0.311 & & 0.239 \\
\hline
\end{tabular}

Table 4. Odds ratios and $95 \%$ confidence intervals of severe IAC risk according to serum GGT quartiles in male AIS patients. Model 1 adjusted for age, smoking, drinking, HT, DM, CHD; Model 2 adjusted for Model 1 plus DBP, TC, TG, Hcys, HbA1c and UA.

the GGT level could not predict the severity of IAC (OR = 1.009, 95\% CI: 0.994-1.024, $\mathrm{p}=0.239)$. Meanwhile, there was no difference in the risk of male patient's calcification between different quantiles groups in both unadjusted and adjusted models (Table 4). Each SD increase of serum GGT levels was associated with risk of severe IAC in univariate analysis $(\mathrm{OR}=1.409,95 \% \mathrm{CI}: 1.091-1.820, P=0.009)$. However, in adjusted models, this association was diminished $(\mathrm{OR}=1.230,95 \% \mathrm{CI}: 0.871-1.735, P=0.239$, Table 4$)$.

\section{Discussion}

This is the first study that assesses the relationship between serum GGT levels and IAC in AIS patients in the Chinese population. In the present study, we found that serum GGT levels were significantly elevated in patients who died. High circulating serum GGT levels in men with AIS are associated with increased all-cause mortality and calcification risk. However, GGT could not independently predict the risk of all-cause mortality and calcification in female patients. In additional, the GGT level could independently predict admission NIHSS scores in male patients with AIS.

Jousilahti et al., identified the relationship between serum GGT levels and alcohol consumption in 14,000 stroke patients ${ }^{10}$. However, the author only interprets GGT as an alternative indicator of alcohol consumption. Emdin M. et al., offers another explanation for the findings based on previous evidence. Although serum GGT is a good indicator of alcohol abuse, epidemiological studies have shown a positive correlation between GGT level and cardiovascular risk factors, regardless of alcohol consumption ${ }^{19}$. A meta-analysis of 926,497 subjects in 10 prospective studies by Zhang X. W. et al. showed that serum GGT level was an independent risk factor for stroke $^{20}$. We found that GGT levels were independently associated with short-term all-cause mortality in men with AIS. Active GGT is present in atherosclerotic plaques ${ }^{21}$. We therefore hypothesized that GGT could be present in cerebral atherosclerotic plaques in patients with AIS. In addition, the GGT levels of patients who died were significantly elevated. Serum GGT level also was independently associated with admission NIHSS score in male patients. We postulated that the GGT enzyme could be associated with the occurrence of an acute stroke event while reflecting the severity of stroke in male.

Several studies have confirmed the relationship between GGT and coronary artery or valve calcification ${ }^{22}$. Cappelli et al. demonstrated that the GGT enzyme was concentrated on lipid deposition and stenosis of aortic valve $^{23}$. IAC is useful as an easily accessible biomarker that reflects the severity of intracranial vascular diseases. Eroglu S. et al., found that serum GGT activity is connected with carotid intima-media thickness (IMT) ${ }^{24}$. The catalytic GGT enzyme was found to be mainly accumulated in atherosclerotic plaques rich in LDL and CD68 ${ }^{+}$ foam cells in cerebral arteries, carotid arteries, and coronary atherosclerotic plaques by endarterectomy ${ }^{21}$. Serum GGT may be involved in the accumulation of GGT in atherosclerotic plaque ${ }^{21}$. GGT can trigger iron-catalyzed LDL oxidation and produce reactive oxygen species, which may promote arterial wall injuries and plaque calcification $^{25}$. In the current study, we report a significant correlation between serum GGT activity and IAC score in male patients. The reference range of GGT activity is similar among different age groups. Although there is significant gender difference, the GGT activity of men is higher than that of the women ${ }^{26}$. We found that each SD increase in male's serum GGT levels was associated with risk of several IAC in univariate analysis. High GGT activity may be an important risk factor for calcification.

As a widely accepted risk factor for stroke patients, Hcys has been broadly used in clinical practice. We first evaluated the relationship between GGT levels and Hcys in patients with AIS. Previous studies have shown that 
combinations of GGT and Hcys can be used to assess early nutritional status in patients with alcohol dependence and heart failure ${ }^{27,28}$. Tu W. J. et al., demonstrated that low circulating levels of retinoic acid (RA) were associated with increased risk of all-cause and CVD mortality in a cohort of patients with first-incidence AIS ${ }^{29}$. Limpach et al., founded that Hcys could inhibit RA synthesis through a specific mechanism for Hcys-induced congenital defects ${ }^{30}$. All-trans retinoic acid increased GGT activity in a cell type-and time-dependent manner ${ }^{31}$. Currently, the relationship among GGT, Hcys and RA could not be determined. But an underlying mechanism may exist between them. In the future, assessment of GGT, Hcys and RA in combinations may be useful to determine the early risk of AIS patients, especially in those with alcohol dependence.

As an observational study, this study has the following shortcomings: First, the number of AIS patients involved in this study is less than other studies. In addition, we only retrospectively reviewed the situations of patients' death at about 60 days; Second, there are currently no suitable quantitative testing, scoring tools and methods for IAC. The risks of IAC and plaque rupture or death in stroke patients remain controversial. To understand if GGT levels could be used as a potential risk factor for CVD patients with cerebrovascular diseases, there is a need for more prospective cohort studies.

\section{Conclusion}

In conclusion, we have evaluated the relationship between serum GGT levels and IAC among AIS patients in the Chinese population. High circulating serum levels of GGT were associated with increased risk of all-cause mortality and IAC in male patients with AIS.

Received: 6 August 2019; Accepted: 13 December 2019;

Published online: 27 December 2019

\section{References}

1. De Silva, D. A. et al. South Asian patients with ischemic stroke: intracranial large arteries are the predominant site of disease. Stroke 38(9), 2592-2594 (2007).

2. Bos, D. et al. Atherosclerotic calcification is related to a higher risk of dementia and cognitive decline. Alzheimer's \& Dementia 11(6), 639-647. e1 (2015).

3. Wu, X. H., Chen, X. Y., Wang, L. J. \& Ka, S. W. Intracranial artery calcification and its clinical significance. J. Clin. Neurol. 12(3), 253-261 (2016).

4. Li, G. et al. Serum gamma-glutamyltransferase levels predict the progression of coronary artery calcification in adults with type 2 diabetes mellitus. Angiology 30(3), 258-262 (2014).

5. Drogan, D. et al. Plasma $\gamma$-glutamyltransferase, cysteinyl-glycine, and oxidized low-density lipoprotein: a pathway associated with myocardial infarction risk? Arterioscler Thromb Vasc. Biol. 30(10), 2053-2058 (2010).

6. Cho, Y. K. et al. Association between serum gamma-glutamyltransferase and the progression of coronary artery calcification. Atherosclerosis 243(1), 300-306 (2015).

7. Atar, A. I. et al. Association between gamma-glutamyltransferase and coronary artery calcification. Int. J. Cardiol. 167(4), 1264-1267 (2013).

8. Lee, D. S. et al. Gamma glutamyl transferase and metabolic syndrome, cardiovascular disease, and mortality risk: the Framingham Heart Study. Arterioscler Thromb Vasc. Biol. 27(1), 127-133 (2007).

9. Ndrepepa, G., Colleran, R. \& Kastrati, A. Gamma-glutamyl transferase and the risk of atherosclerosis and coronary heart disease. Clin. Chim. Acta. 476(1), 130-138 (2018).

10. Jousilahti, P., Rastenyte, D. \& Tuomilehto, J. Serum gamma-glutamyl transferase, self-reported alcohol drinking, and the risk of stroke. Stroke 31(8), 1851-1855 (2000).

11. Gurbuzer, N., Gozke, E. \& Ayhan, B. Z. Gamma-glutamyl transferase levels in patients with acute ischemic stroke. Cardiovasc. Psychiatry. Neurol. 170626 (2014).

12. Tu, W. J. et al. $\gamma$-Glutamyl transferase as a risk factor for all-cause or cardiovascular disease mortality among 5912 ischemic stroke. Stroke 48, 2888-2891 (2017).

13. Gang, L. et al. Serum gamma-glutamyltransferase levels predict the progression of coronary artery calcification in adults with type 2 diabetes mellitus. Angiology 30, 258-262 (2014).

14. Adams, H. P. Jr. et al. for the TOAST Investigators. Classification of subtype of acute ischemic stroke: definitions for use in a multicenter clinical trial. Stroke 24, 35-41 (1993).

15. Kim, J. M. et al. Intracranial Arterial Calcificationes Can Reflect Cerebral Atherosclerosis Burden. J. Clin. Neurol. 15(1), 38-45 (2019).

16. Olatunji, R. B., Adekanmi, A. J. \& Ogunseyinde, A. O. Intracranial arterial calcification in black Africans with acute ischaemic stroke. Cerebrovasc. Dis. Extra. 8, 26-38 (2018).

17. Babiarz, L. S., Yousem, D. M., Bilker, W. \& Wasserman, B. A. Middle cerebral artery infarction: relationship of cavernous carotid artery calcification. AJNR. Am. J. Neuroradiol. 26, 1505-1511 (2005).

18. Mak, H. K. et al. Computed tomography evaluation of intracranial atherosclerosis in Chinese patients with transient ischaemic attack or minor ischaemic stroke-its distribution and associated vascular risk factors. J. Stroke Cerebrovasc. Dis. 18, 158-163 (2009).

19. Emdin, M. et al. Serum gamma-glutamyltransferase as a risk factor of ischemic stroke might be independent of alcohol consumption. Stroke 33(4), 1163-1164 (2002).

20. Zhang, X. W. et al. Association between gamma-glutamyltransferase level and risk of stroke: a systematic review and meta-analysis of prospective studies. J. Stroke. Cerebrovasc. Dis. 24(12), 2816-2823 (2015).

21. Emdin, M., Pompella, A. \& Paolicchi, A. Gamma-glutamyltransferase, atherosclerosis, and cardiovascular disease: triggering oxidative stress within the plaque. Circulation 112, 2078-2080 (2005).

22. Bozbas, H. et al. Serum gamma-glutamyltransferase activity is increased in patients with calcific aortic valve stenosis. J. Heart. Valve. Dis. 17(4), 371-375 (2008).

23. Cappelli, S. et al. Aortic valve disease and gamma-glutamyltransferase: accumulation in tissue and relationships with calcific degeneration. Atherosclerosis 213(2), 385-391 (2010).

24. Eroglu, S. et al. Association between serum gamma-glutamyltransferase activity and carotid intima-media thickness. Angiology 62(2), 107-110 (2011).

25. Paolicchi, A. et al. Gamma-glutamyl transpeptidase-dependent iron reduction and low density lipoprotein oxidation: a potential mechanism in atherosclerosis. J. Invest. Med. 47, 151-160 (1999).

26. Kunutsor, S. K. Gamma-glutamyltransferase-friend or foe within? Liver. Int. 36, 1723-1734 (2016). 
27. Rinck, D. et al. Combinations of carbohydrate-deficient transferrin, mean corpuscular erythrocyte volume, gammaglutamyltransferase, homocysteine and folate increase the significance of biological markers in alcohol dependent patients. Drug Alcohol Depend 89(1), 60-65 (2007).

28. Makarewicz-Wujec, M. \& Kozlowska-Wojciechowska, M. Nutrient intake and serum level of gamma-glutamyltransferase, MCP-1 and homocysteine in early stages of heart failure. Clin. Nutr. 30(1), 73-78 (2011).

29. Tu, W. J. et al. Lower serum retinoic acid level for prediction of higher risk of mortality in ischemic stroke. Neurology 92(15), e1678-e1687 (2019).

30. Limpach, A., Dalton, M. \& Miles, R. Homocysteine inhibits retinoic acid synthesis: a mechanism for homocysteine-induced congenital defects. Exp. Cell. Res. 260(1), 166-174 (2000).

31. El. Yaagoubi, M., Hachad, H., Leh, H., Siest, G. \& Wellmanet, M. Gamma-glutamyltransferase expression during all-trans retinoic acid-induced differentiation of hematopoietic cell lines. FEBS letters 369(2-3), 183-186 (1995).

\section{Acknowledgements}

The present study was supported by grants from the Hospital Talents Support Fund of Hubei Provincial Hospital of TCM (201612022B) and Natural Science Foundation of Hubei Province of China (2018CFB699). The authors are indebted to the other investigators, staff, and participants for expert technical assistance.

\section{Author contributions}

G.L. and Y.B.D. contributed to the conception and design of the study. T.Y. and J.L. recruited the subjects. Fellow-up patients were performed by Q.L. and Q.C. T.Y. analyzed the data and wrote the initial draft of the paper. Z.C.L. participated in its design and supervised the study. All authors contributed to the writing, reviewing, and revising of the manuscript.

\section{Competing interests}

The authors declare no competing interests.

\section{Additional information}

Correspondence and requests for materials should be addressed to G.L. or Y.D.

Reprints and permissions information is available at www.nature.com/reprints.

Publisher's note Springer Nature remains neutral with regard to jurisdictional claims in published maps and institutional affiliations.

(c) (i) Open Access This article is licensed under a Creative Commons Attribution 4.0 International

License, which permits use, sharing, adaptation, distribution and reproduction in any medium or format, as long as you give appropriate credit to the original author(s) and the source, provide a link to the Creative Commons license, and indicate if changes were made. The images or other third party material in this article are included in the article's Creative Commons license, unless indicated otherwise in a credit line to the material. If material is not included in the article's Creative Commons license and your intended use is not permitted by statutory regulation or exceeds the permitted use, you will need to obtain permission directly from the copyright holder. To view a copy of this license, visit http://creativecommons.org/licenses/by/4.0/.

(C) The Author(s) 2019 